\title{
A 20-Year-Old Retained Surgical Gauze Mimicking a Spinal Tumor: A Case Report
}

\author{
Sungjoon Lee ${ }^{1}$, Bomi Kim², Jung Soo Kim¹, Byeong Sam Choi ${ }^{1}$ \\ Departments of ${ }^{l}$ Neurosurgery and ${ }^{2}$ Pathology, Inje University Haeundae Paik Hospital, Busan, Korea
}

\begin{abstract}
A 79-year-old man visited our clinic complaining of lower back and left leg radiating pain that began 1 month prior to his presentation. He underwent surgery for lumbar disc herniation 20 years ago at another hospital. Magnetic resonance imaging revealed left-sided foraminal stenosis at $L 4-5$. In addition, a paraspinal mass occupying the $L 4$ spinous process and left lamina was observed. We subsequently performed an L4-5 decompression and fusion. During the operation, retained surgical gauze with granulation tissue was found. The term gossypiboma is used to define a mass lesion consisting of retained surgical gauzes and an adjacent foreign body reaction. Gossypibomas are uncommon in the paraspinal area and are mostly asymptomatic in chronic cases. Because there are no specific clinical or radiological signs, they can be confused with other tumorous conditions. Gossypibomas should be included in the differential diagnosis of paraspinal soft-tissue masses detected in patients with a history of prior spinal surgery.
\end{abstract}

Key Words: Foreign bodies $\cdot$ Gossypiboma $\cdot$ Textiloma $\cdot$ Retained surgical gauze $\cdot$ Spinal surgery $\cdot$ Spinal tumor

\section{INTRODUCTION}

Gossypiboma is a term used to describe masses consisting of retained cotton surgical gauzes and an adjacent foreignbody reaction ${ }^{1,2,5-7,9)}$. Its radiologic features are well-known because there are many reports in the literature regarding this phenomenon following abdominal and pelvic surgeries, ${ }^{5,10,11)}$. Surgical gauzes usually contain radiopaque filaments or markers that enable the diagnosis of retained surgical gauze. However, without a radiopaque marker, the diagnosis of gossypiboma in spine surgery is rather difficult because of its low incidence in the paraspinal location; it may be confused with soft tissue tumors ${ }^{2,7,9,13,15)}$.

In this report, we describe the case of an asymptomatic retained surgical gauze discovered 20 years after lumbar disc surgery. In addition, relevant literature is reviewed regarding the clinical presentation, radiological findings, and differential diagnosis.

\section{CASE REPORT}

A 79-year-old man visited our clinic complaining of lower

- Received: May 3, 2016 • Revised: July 19, 2016

- Accepted: July 25, 2016

Corresponding Author: Byung Sam Choi

Department of Neurosurgery, Inje University, Haeundae Paik Hospital, 875 Haeun-daero, Haeundae-gu, Busan 48108, Korea

Tel: +82-51-797-0241, Fax: +82-51-797-0841

E-mail: ttt333@hanmail.net

$\otimes$ This is an open access article distributed under the terms of the Creative Commons Attribution Non-Commercial License (http://creativecommons.org/licenses/by-nc/4.0/) which permits unrestricted non-commercial use, distribution, and reproduction in any medium, provided the original work is properly cited.
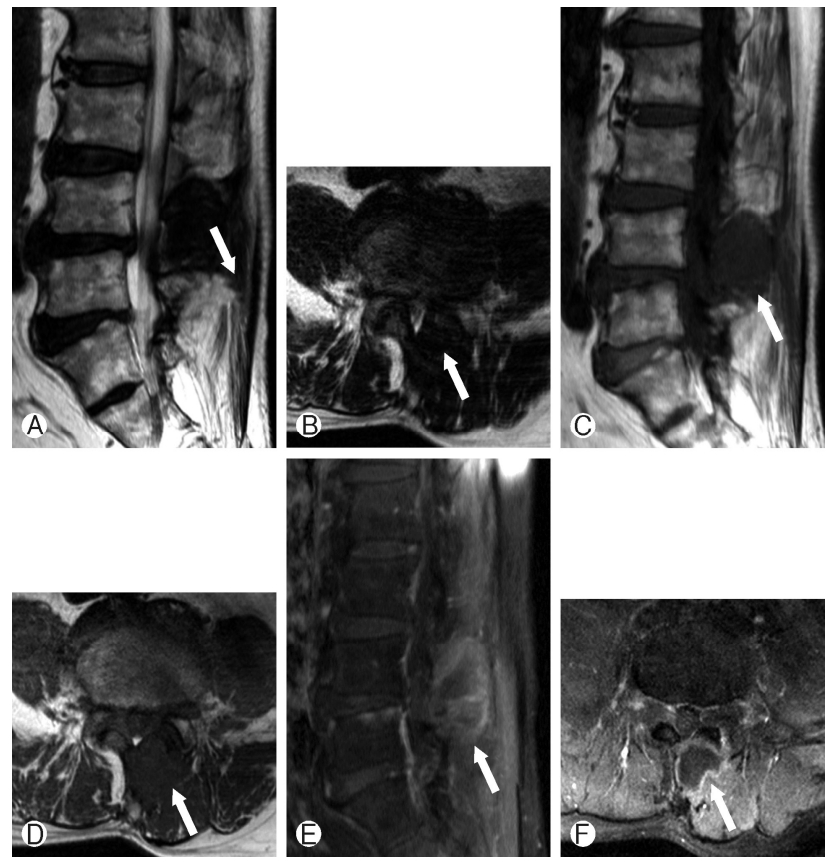

Fig. 1. Preoperative magnetic resonance imaging. In each image, the mass lesion is indicated with a white arrow. (A), (B) T2-weighted sagittal and axial images. A $4.3 \times 2.7 \times 2.3-\mathrm{cm}$ sized mass lesion showing low and heterogeneous signal intensity occupies the L4 spinous process and left lamina. (C), (D) T1-weighted sagittal and axial images. The mass shows an iso- to hypointense signal compared with the surrounding musculature. (E), (F) T1weighed sagittal and axial images after gadolinium injection. Only the peripheral rim of the mass enhances. 
back pain and left leg radiating pain that began a month prior to presentation. He underwent surgery for lumbar disc herniation 20 years ago at another hospital. Upon neurological examination, no motor weakness was found, and hypoesthesia and radiating pain were noted at his left anterior thigh. His body temperature at admission was $36.3^{\circ} \mathrm{C}$. On laboratory examination, his white blood cell count was within the normal range $(6,540 / \mu \mathrm{L}$; normal reference range, $4,000-10,000 / \mu \mathrm{L})$, and his C-reactive protein level was normal $(0.14 \mathrm{mg} / \mathrm{dL}$; normal reference range, $0-0.5 \mathrm{mg} / \mathrm{dL}$ ). Magnetic resonance imaging (MRI) revealed left foraminal stenosis at L4-5 (Fig. 1). A $4.3 \times 2.7 \times 2.3-\mathrm{cm}$-sized mass lesion occupying the L4 spinous process and the left lamina was observed, showing low and heterogeneous signal intensity on T2-weighted imaging (Fig. 1A, B). It was iso- to hypointense compared with the surroun- ding musculature on T1-weighted imaging (Fig. 1C, D). Only its outer rim was enhanced after gadolinium injection (Fig. 1E, F). Computed tomography (CT) showed an osteolytic mass with sharp margins (Fig. 2). A benign bone tumor arising from the lamina, such as osteoblastoma, was our initial diagnosis. We assumed that the left foraminal stenosis at L4-5 was the cause of the patient's left leg pain and that the mass lesion, causing no symptoms, was incidental.

The mass was removed under general anesthesia, and an L4-5 facetectomy and interbody fusion was performed. During the operation, retained surgical gauze with heavy granulation tissue was found (Fig. 3). The surgical gauze was made of cotton, and it was hardened and well encapsulated with fi-

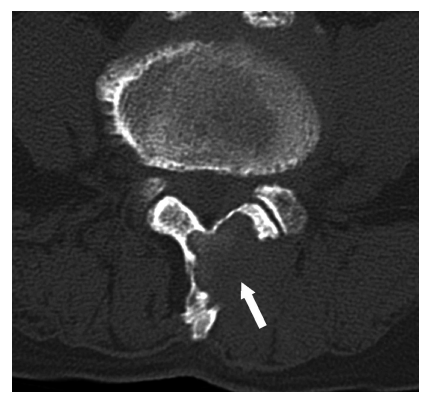

Fig. 2. Computed tomography scan showing a low attenuated osteolytic mass with sharp margins (white arrow).

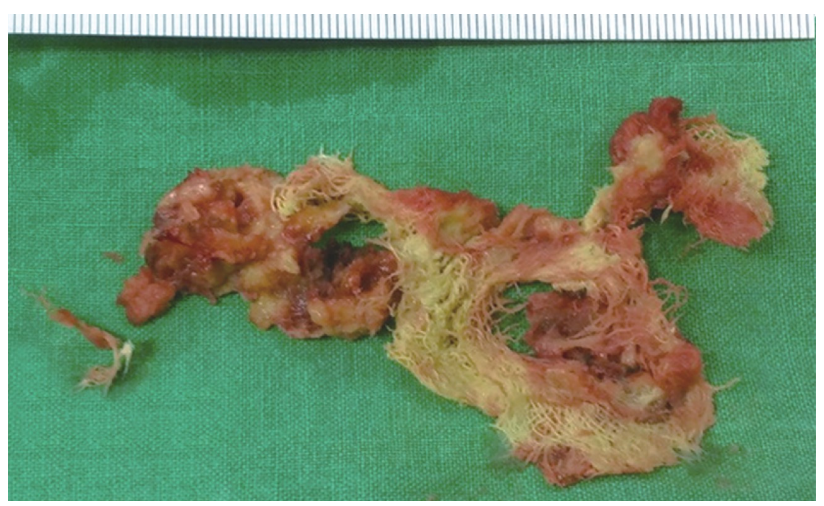

Fig. 3. Intraoperative photo of the mass; a retained surgical gauze. brotic tissue. There were no signs of infection, such as pus discharge or abscess formation. We excised the mass completely, along with the surrounding fibrotic capsule. Histopathological examination revealed suture material with granulation tissue (Fig. 4). There were no unusual events during the postoperative period. The patient's back and left leg pain had almost completely resolved at his 6-month postoperative follow-up.

\section{DISCUSSION}

Although rare, retained surgical gauzes inside of a patient's body can occur in various surgical fields. Gossypiboma is more common in abdomino-pelvic surgeries than in surgeries in other parts of the body, including the extremities and paraspinal areas $^{216}$. There is much dead space in the abdominal and pelvic cavity, which may limit the surgeon's visual field. Therefore, surgical gauzes that are hidden in those spaces are prone to being lost and are discovered after the surgery. Because there is little or no dead space in the operative field of spinal surgery, it is not surprising that gossypibomas in the paraspinal area have a significantly lower incidence than those in the abdominal and pelvic regions.

Gossypibomas are subclassified into an acute and a chronic form ${ }^{2,49}$. In the acute form, exudative reactions dominate. Formation of abscesses and fistulous tracts to the skin surface frequently occur, and patients usually become symptomatic in the early postoperative period ${ }^{24}$. The chronic form is characterized by a well-encapsulated aseptic foreign body granuloma, with no symptoms or non-specific subjective symptoms $^{2,4)}$. Surgical gauzes made of cotton are inert; they do not activate specific biochemical reactions other than slow foreign-body reactions characterized by granuloma formation 24,9). Thus, most chronic gossypibomas cause few or no symptoms. In our case, it was the chronic form of gossypiboma which remained inert in patient's body for 20 years without any symptoms. Because it was made of cotton which was inert, we presume that it could remain asymptomatic not stimulating
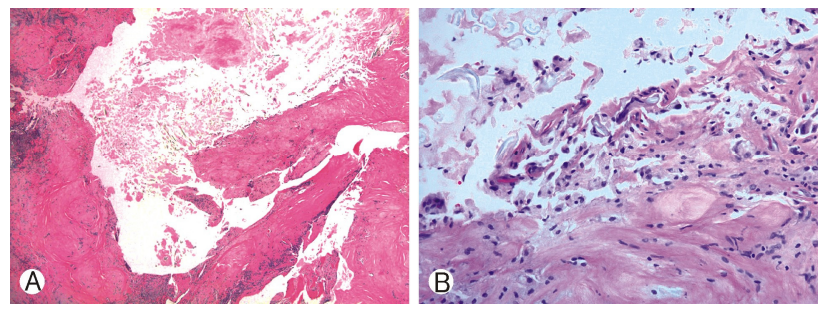

Fig. 4. (A) Low power views revealed a foreign body granuloma with cystic changes. In the solid area, there are amorphous materials that resemble degenerated gauze. Necrosis and suture fibers are noted in the lumen of the cyst $(H \& E, \times 40)$. (B) In the foreign body granuloma, there are many histiocytes, including foam cells and multinucleated giant cells. Red arrow points to the fused macrophage, which phagocytized suture material ( $\mathrm{H} \&$ $\mathrm{E}, \times 400)$. 
active biochemical reactions and inflammations.

The radiologic features of gossypibomas in the abdominal cavity are well-documented ${ }^{5,10,11)}$. If the gauze contains a radiopaque barium sulfate marker, it can be easily identified and diagnosed with simple X-ray imaging. Without the marker, as shown in the present case, the level of difficulty in making the diagnosis of gossypiboma skyrockets. In addition, only approximately 50 cases have been reported in the paraspinal location $^{1,2,4,6,7,9,13,15)}$, and most of the literature has described varying radiologic features in these cases. However, there are some salient radiologic features that can be used to diagnose a gossypiboma.

On CT, the most common characteristic feature of a gossypiboma is the spongiform pattern formed by gas bubbles ${ }^{2,8,14}$. However, the gas bubbles are absorbed as time passes; thus, this typical pattern is not found in the chronic form ${ }^{2)}$. In the paraspinal region, gossypibomas showing osteolytic changes with marginal sclerosis on CT have been reported ${ }^{2,7,12)}$. Our case also showed similar CT findings, which may be specific features of chronic gossypibomas.

On MRI, gossypibomas appear as well-defined masses with fibrous capsules that exhibit low signal intensity on T1-weighted imaging, high central signal intensity with a hypointense rim on T2-weighted imaging and strong peripheral enhancement on contrast-enhanced images ${ }^{6,9)}$. However, gossypibomas appear to have variable magnetic resonance signal intensity that depends on the amount of fluid and protein associated with the lesion ${ }^{6}$, and there have been many reports that have shown MRI findings other than those described above $\mathrm{e}^{2,3,7,9,12)}$. The major difference arises from T2-weighted images characterized by heterogeneous signals composed of mainly dark signals. Because gauze fibers appear as low intensity strips on T2-weighted imaging ${ }^{6}$, this may be a characteristic MRI finding of gossypiboma. In the present case, the mass showed low and heterogeneous signal intensity on T2-weighted imaging and iso- to hypointense signal intensity on T1-weighted imaging with peripheral rim enhancement after gadolinium injection as presented in the literature ${ }^{27,9)}$. When we reviewed this case retrospectively, we concluded that it may not have been impossible to make a positive radiologic diagnosis.

Most reports have demonstrated that the clinical symptoms associated with gossypibomas were well treated by complete removal of the masses $1,2,4,7,9,12,13,15)$. However, the clinical symptoms associated with gossypibomas may not be prominent, especially in chronic gossypibomas. Therefore, careful assessments of the patient's symptoms are essential. If the gossypiboma appears to be asymptomatic, the potential risk of evolution of the gossypiboma and the risk of surgical removal must be weighed before choosing the treatment strategy. In the present case, the gossypiboma was asymptomatic. The cause of the patient's symptoms was the left foraminal stenosis at L4-5. Our diagnostic impression of the mass was an osteoblastoma, and we intended to remove it. Because the mass was located within the surgical corridor for decompression and fusion, we were able to minimize harm to the patient despite our incorrect preoperative diagnosis.

After all, gossypiboma is the result of surgical error. The importance of not leaving surgical gauzes inside patients' bodies after surgery cannot be overemphasized. Surgical gauzes must be counted before closing patients' wounds and at the end of surgery. In addition, gauzes with radiopaque markers should be used to easily identify retained surgical gauzes.

\section{CONCLUSION}

Paraspinal gossypibomas are uncommon and are thus sometimes difficult to diagnose. Most chronic gossypibomas are indolent and do not show specific clinical or radiologic signs, making the diagnosis even more difficult. If the patient has a history of previous spinal surgery, and the mass is located at the previous surgical site, gossypiboma should be included in the differential diagnosis. With reasonable suspicion and the help of some characteristic radiologic features demonstrated in the literature, making a correct diagnosis may not be an unfeasible task.

\section{CONFLICT OF INTEREST}

No potential conflict of interest relevant to this article was reported.

\section{REFERENCES}

1. Atabey C, Turgut M, Ilica AT: Retained surgical sponge in differential diagnosis of paraspinal soft-tissue mass after posterior spinal surgery: report of eight cases. Neurol India 57:320-323, 2009

2. Aydogan M, Mirzanli C, Ganiyusufoglu K, Tezer M, Ozturk I: A 13-year-old textiloma (gossypiboma) after discectomy for lumbar disc herniation: a case report and review of the literature. Spine J 7:618-621, 2007

3. Hakan T, Aydoseli A, Demir K, Aker F: Clinical, pathological and radiological features of paraspinal textiloma: report of two cases and review of the literature. Neurol Neurochir Pol 43: 475-478, 2009

4. Is M, Karatas A, Akgul M, Yildirim U, Gezen F: A retained surgical sponge (gossypiboma) mimicking a paraspinal abscess. Br J Neurosurg 21:307-308, 2007

5. Kim CK, Park BK, Ha H: Gossypiboma in abdomen and pelvis: MRI findings in four patients. AJR Am J Roentgenol 189:814817,2007

6. Kim HS, Chung TS, Suh SH, Kim SY: MR imaging findings of paravertebral gossypiboma. AJNR Am J Neuroradiol 28:709713, 2007

7. Kobayashi T, Miyakoshi N, Abe E, Abe T, Suzuki T, Takahashi $\mathrm{M}$, et al: Gossypiboma 19 years after laminectomy mimicking a malignant spinal tumour: a case report. J Med Case Rep 8:311, 2014

8. Kopka L, Fischer U, Gross AJ, Funke M, Oestmann JW, Grabbe E: CT of retained surgical sponges (textilomas): pitfalls in detection and evaluation. J Comput Assist Tomogr 20:919-923, 1996 
9. Kucukyuruk B, Biceroglu H, Abuzayed B, Ulu MO, Kafadar AM: Paraspinal gossybipoma: A case report and review of the literature. J Neurosci Rural Pract 1:102-104, 2010

10. Murphy CF, Stunell H, Torreggiani WC: Diagnosis of gossypiboma of the abdomen and pelvis. AJR Am J Roentgenol 190: W382, 2008

11. O'Connor AR, Coakley FV, Meng MV, Eberhardt SC: Imaging of retained surgical sponges in the abdomen and pelvis. AJR Am J Roentgenol 180:481-489, 2003

12. Rohde V, K?ker W, Gilsbach JM: Foreign body granuloma mimicking a benign intraspinal tumour. Br J Neurosurg 13:417- 419, 1999
13. Sahin S, Atabey C, Simşek M, Naderi S: Spinal textiloma (gossypiboma): a report of three cases misdiagnosed as tumour. Balkan Med J 30:422-428, 2013

14. Sheward SE, Williams AG Jr, Mettler FA Jr, Lacey SR: CT appearance of a surgically retained towel (gossypiboma). J Comput Assist Tomogr 10:343-345, 1986

15. Turgut M, Akyüz O, Ozsunar Y, Kacar F: Sponge-induced granuloma ("gauzoma") as a complication of posterior lumbar surgery. Neurol Med Chir (Tokyo) 45:209-211, 2005

16. Van Goethem JW, Parizel PM, Perdieus D, Hermans P, de Moor J: MR and CT imaging of paraspinal textiloma (gossypiboma). J Comput Assist Tomogr 15:1000-1003, 1991 